\title{
El boletín Faraday (1928-29) y las relaciones entre historia $y$ didáctica de las ciencias
}

\section{The bulletin Faraday (1928-29) and the relations between history and didactics of science}

Luis Moreno Martínez

Instituto Interuniversitario López Piñero, Universitat de València (España)

luis.moreno-martinez@uv.es

RESUMEN - Faraday ha sido considerada una de las primeras publicaciones especializadas en didáctica e historia de la ciencia en España. Sin embargo, hasta la fecha su recuperación y estudio se revelaba pendiente. A través del análisis inédito de esta publicación de principios del siglo xx, se abordan las relaciones entre historia y didáctica de las ciencias desde una mirada diacrónica. Dicho análisis ha puesto de manifiesto cómo la didáctica de las ciencias contribuyó a impulsar la forja disciplinar de la historia de la ciencia y cómo esta última nutrió a la didáctica de las ciencias de un amplio sustrato metodológico y epistemológico. Este análisis también ha ofrecido varios elementos de reflexión para pensar críticamente retos de la actual didáctica de las ciencias experimentales, tales como su conexión con el profesorado y los usos didácticos de la historia de la ciencia.

PALABRAS CLAVE: Historia de la ciencia; Didáctica de las ciencias experimentales; Enseñanza de las ciencias; Disciplinas académicas; Revistas pedagógicas.

ABSTRACT - Faraday has been pointed out as one of the first specialized journals on the didactics and history of science in Spain. Starting from the analysis of this unstudied journal, this paper examines the relations between history and didactics of science in the early twentieth-century Spain. I argue that didactics of science was a key factor in the emergence of history of science as an academic discipline. Likewise, history of science provided didactics of science with a wide range of methodological and epistemological resources. Besides, the analysis of Faraday has highlighted several reflection elements for thinking critically about the relations between didactics of science and science teachers, and the didactic uses of the history of science.

KEYWORD: History of science; Didactics of science; Science teaching; Academic disciplines; Pedagogy journals. 


\section{OBJETIVOS, MARCO TEÓRICO Y APROXIMACIÓN METODOLÓGICA}

El presente artículo indaga en las relaciones entre historia y didáctica de las ciencias a través de una publicación que, pese a haber sido señalada como la «primera revista española dedicada a la didáctica e historia de la ciencia» (Checa Godoy, 2002, p. 243), no ha sido objeto de recuperación ni análisis hasta ahora: el boletín Faraday. Mediante el análisis inédito de esta publicación se pretenden tres objetivos de investigación. En primer lugar, contribuir a recuperar esta publicación científico-pedagógico pionera, dado su olvido generalizado tanto en el seno de la historiografía científico-educativa como en la propia genealogía del colectivo didacta. En segundo lugar, se analiza el papel que la didáctica de las ciencias tuvo en el impulso disciplinar de la historia de la ciencia. En tercer lugar se estudia el papel desempeñado por la historia de la ciencia en el desarrollo de la didáctica de las ciencias experimentales.

El estudio de las relaciones entre historia y didáctica de las ciencias se inscribe en un marco cronológico de gran interés analítico: el primer tercio del siglo xx. Al tratarse de un periodo en el que ambas disciplinas estaban lejos de ostentar su carácter disciplinar actual, su análisis ofrece un proscenio privilegiado para pensar conexiones que no necesariamente encontraremos en las disciplinas actuales. Ello permitirá así evitar la visión teleológica que destaca de este "periodo adisciplinar» solo aquellos elementos que después vertebraron las respectivas disciplinas. Asimismo, al abandonar el "periodo disciplinar» es posible abordar un análisis que huya de una visión internalista del desarrollo de la disciplina. Se trata de miradas analíticas en clara consonancia con la actual historia social y cultural del conocimiento (Burke, 2017), las nuevas perspectivas historiográficas en historia de la ciencia (Simon y Herrán, 2008), la historia de la ciencia promovida desde la investigación didáctica (Quintanilla Gatica et al., 2014) y las pautas metodológicas sobre análisis diacrónico de cuestiones actuales en educación científica (Rudolph, 2019). Sin embargo, para acometer dichos objetivos, se hace fundamental una previa reflexión sobre la propia concepción de disciplina en la que se enmarca el análisis.

Las disciplinas académicas del presente pueden entenderse como constructos en una constante tensión entre pasado y futuro. En el marco de la sociología del campo intelectual de Bourdieu, es posible concebir las disciplinas científicas como territorios propios cuyos miembros integrantes comparten lenguajes, valores, instituciones y una serie de problemas no resueltos (Nye, 1993), entre otros elementos vertebradores. $\mathrm{Al}$ igual que los Estados nación, una disciplina requiere del reconocimiento externo de otros «territorios» a fin de lograr legitimidad en su correspondiente campo del saber. La interacción con otras disciplinas constituye así un aspecto de gran relevancia para su desarrollo (Gavroglu y Simoes, 2012), algo que Faraday ilustra para el caso de la didáctica y la historia de las ciencias. Precisamente, la historia constituye otro elemento vertebrador de las disciplinas, actuando a modo de genealogía que conecta a los integrantes del presente con tradiciones y saberes pretéritos de los que se erigen legítimos descendientes, a los que también cuestionan y en ocasiones silencian. Si estas genealogías conectan la disciplina con el pasado, la enseńanza la conecta con el futuro; motivo por el cual la selección y organización de saberes que acarrea la enseñanza ha sido señalada como un factor clave en la forja de las propias disciplinas científicas, que tendrían así un origen didáctico (Hannaway, 1975). Así, los futuros miembros de las disciplinas han de ser instruidos en los valores, prácticas, lenguajes y saberes de esta a fin de garantizar su continuidad. La didáctica de las ciencias experimentales ilustra todos estos elementos definitorios de una disciplina académica.

La didáctica de las ciencias experimentales es, no obstante, una disciplina joven en comparación con la dilatada tradición de las ciencias de cuya enseñanza-aprendizaje se ocupa, las cuales gestaron su conformación disciplinar a lo largo del siglo XIx. Desde esta perspectiva es posible asumir que la reflexión epistemológica sobre el desarrollo histórico de las ciencias sea mucho más amplia, diversificada y problematizada que la historia de su didáctica. No obstante, desde finales del siglo xx, varios didactas de las ciencias (o didactólogos) asumieron de forma pionera el estudio del pasado de su disciplina. 
En este terreno cabe destacar los trabajos de Porlán Ariza (1998), Adúriz Bravo e Izquierdo Aymerich (2002) para la disciplina en Espańa. Así, en un primer esfuerzo por abordar el pasado de la entonces emergente didáctica de las ciencias experimentales, se distinguieron varias etapas en su desarrollo histórico. Para el contexto español, en el que se inscribe el presente trabajo, se apuntó la existencia de una primera etapa adisciplinar (desde finales del siglo xix hasta mediados del siglo xx) caracterizada por la producción discontinua de trabajos sobre enseñanza de las ciencias; una etapa tecnológica (localizable en las décadas de 1950 y 1960) definida por el interés en el estudio de las reformas curriculares; una etapa protodisciplinar (situable en la década de 1970) en la que se produjeron los primeros consensos en torno a cuestiones como la importancia de las concepciones alternativas del alumnado en la enseñanza-aprendizaje de las ciencias; una etapa emergente (que abarcaría las décadas de 1980 y 1990) en la que los cada vez mayores consensos teóricos y metodológicos en torno al constructivismo se articularon en una creciente estructura académica universitaria; y la etapa actual, en la que la didáctica de las ciencias constituye una disciplina consolidada caracterizada por modelos genuinamente didácticos y una amplia presencia universitaria (Porlán Ariza, 1998; Adúriz Bravo, 2000; Adúriz Bravo e Izquierdo Aymerich, 2002).

Estos primeros trabajos contribuyeron a dotar a la didáctica de las ciencias experimentales de un pasado. Las propias ciencias experimentales también produjeron dichas narrativas genealógicas sobre su pasado a lo largo de su consolidación durante el siglo xIx. Estas narrativas fueron producidas por los propios científicos, hoy conocidos como científicos-historiadores. Las narrativas históricas de estos científico-historiadores sirvieron de sustrato para los análisis producidos por la primera generación de historiadores de la ciencia. La historia de la ciencia tuvo su forja disciplinar en las primeras décadas del siglo xx. Por ello, a lo largo del siglo xx las ciencias experimentales han visto ampliadas, matizadas y problematizadas sus primeras narrativas históricas (Kragh, 2007). Se ha transitado así de una historia netamente genealógica basada en estadios o etapas y centrada en el desarrollo interno de las disciplinas científicas (la producida por el científico-historiador) a una historia social, cultural y material de las ciencias (la producida por el historiador de la ciencia) (Bertomeu Sánchez y García Belmar, 2008; Nieto-Galan, 2014). Todo ello permite trazar un paralelismo en el tiempo entre la producción de nuevas narrativas históricas y el nivel de maduración de las propias disciplinas. Esta misma situación es identificable para el ámbito de la didáctica de las ciencias experimentales. Así, trazadas las primeras genealogías disciplinares, en las últimas décadas se han producido nuevas narrativas históricas.

Desde esta óptica, Bernal Martínez (2001) señaló la necesidad de una historia de la didáctica de las ciencias experimentales que incorporase personajes, espacios y experiencias escolares que hasta entonces habían recibido una escasa atención por parte de la disciplina. Progresivamente, la historia de la didáctica de las ciencias experimentales fue experimentado una notable expansión en el ámbito académico, atrayendo la atención tanto de historiadores de la ciencia interesados en las aulas (Bertomeu Sánchez, 2016) como de historiadores de la educación interesados en la ciencia escolar (Viñao, 2004). En esta línea, las primeras dos primeras décadas del siglo xxi han sido testigo de una profunda renovación, consolidación y diversificación de los estudios históricos sobre ciencia en las aulas (Kaiser, 2005; Olesko, 2006, 2014), habiéndose afirmado que «no parece que la historia de la ciencia pueda prescindir de la historia de la educación científica» (Nieto Galán, 2011, p. 203).

La didáctica de las ciencias experimentales ha mantenido desde su consolidación disciplinar una estrecha relación con la historia de la ciencia, trazable al ser posible localizar múltiples trabajos sobre historia y epistemología de las ciencias en publicaciones propias de la disciplina. En este ámbito es sobresaliente el caso de la revista Enseñanza de las Ciencias, que incluyó en su primer número un artículo reivindicando la importancia de la historia de la ciencia en la enseñanza (Navarro Brotons, 1983). La propia investigación didáctica ha puesto de manifiesto la necesidad de que la historia escolar de la ciencia sea aquella que parta del trabajo del historiador de la ciencia, adaptándolo a las necesidades del 
profesorado en un marco avalado por la investigación didáctica (Quintanilla Gatica et al., 2014). Esta misma premisa se erige como fundamental no solo para la producción y uso de narrativas escolares sobre historia de la ciencia, sino también para la construcción de la propia historia de la didáctica de las ciencias experimentales y su incorporación a la reflexión epistemológica en el seno de la disciplina. Se trata de una línea de investigación que, si bien mucho menos transitada que el estudio de los usos didácticos de la historia de la ciencia, ha experimentado un notable impulso en los últimos años en el ámbito internacional. En este punto cabe destacar los trabajos de Olesko (2014) sobre la categorización de la enseñanza de las ciencias para la investigación histórica y los análisis de Rudolph (2019) sobre el análisis diacrónico de cuestiones didácticas actuales en la enseñanza de las ciencias. Se trata, asimismo, de una línea de investigación que exige miradas metodológicas necesariamente interdisciplinares, ampliamente asumibles en el seno de la didáctica de las ciencias experimentales, desde el cual ya se señaló hace décadas la "apertura interdisciplinar» como una muestra de consolidación que en el futuro debía caracterizar a la disciplina (Porlán Ariza, 1998, p. 182).

Desde esta «apertura interdisciplinar» y partiendo del alto valor de la historia para comprender, conocer, saber y relacionar en la enseñanza de las ciencias (Izquierdo Aymerich, 2011), se ha analizado Faraday, atendiendo a dos miradas metodológicas propias de la interfase entre el trabajo del didacta y del historiador de la ciencia. En primer lugar, se han adoptado las pautas propias de la investigación histórica a fin de identificar los elementos definitorios de esta publicación pedagógico-científica. El olvido de Faraday en la literatura académica ha hecho necesario acometer un trabajo de descripción de la publicación (primer objetivo de investigación) antes de abordar el análisis de las relaciones entre historia y didáctica de las ciencias (segundo y tercer objetivo). La búsqueda, recuperación y análisis de los ejemplares de Faraday ha sido posible gracias al catálogo de la Biblioteca de Catalunya, donde se conservan 12 de los 13 ejemplares localizados. El examen realizado ha mostrado que se trató de una publicación de periodización cuasimensual, con la excepción del periodo estival, cuyo primer número fue publicado en enero de 1928. Tal y como se pude observar en la tabla 1, el último número localizado corresponde a mayo de 1929. Como se podrá comprobar en el próximo epígrafe, la descripción de Faraday se ha llevado a cabo atendiendo a los elementos definitorios propios de publicaciones pedagógicas de la época, como la Revista de Escuelas Normales (Díez Torre et al., 1988) o Escuela Moderna (Montes Moreno, 2003). Entre estos elementos se incluyen el editor, los objetivos de la publicación y su estructura, a la que hay que ańadir un elemento revelado como clave en el marco de la historiografía actual de las ciencias: los públicos (Nieto-Galan, 2011). Como se verá a través de las páginas de Faraday, lejos de meros receptores pasivos, los públicos han desempeñado un papel activo que ha condicionado la circulación de saberes científicos y pedagógicos.

Tabla 1.

Números del boletín Faraday publicados y localizados (1928-1929)

\begin{tabular}{|c|l|c|l|l|l|}
\hline Núm. & \multicolumn{1}{|c|}{ Mes / año } & Núm. & \multicolumn{1}{|c|}{ Mes / año } & Núm & \multicolumn{1}{c|}{ Mes / año } \\
\hline 1 & Enero /1928 & 6 & Septiembre / 1928 & 11 & Marzo / 1929 \\
\hline 2 & Febrero / 1928 & 7 & Octubre / 1928 & 12 & Abril / 1929 \\
\hline 3 & Marzo / 1928 & 8 & Nov.-Dic. / 1928 & 13 & Mayo / 1929 \\
\hline 4 & Abril / 1928 & 9 & Enero / 1928 & $\begin{array}{l}\text { Números localizados: todos } \\
\text { excepto el número de abril } \\
\text { de 1928 }\end{array}$ \\
\hline 5 & Mayo / 1928 & 10 & Febrero, / 1929 \\
\hline
\end{tabular}


En segundo lugar, la consecución de los otros dos objetivos de la investigación ha sido posible gracias a una aproximación metodológica cimentada en tres estadios fundamentales. Primero se ha contextualizado la publicación del boletín en el marco histórico correspondiente, a fin de evitar presentismos y anacronismos. Así, la abundante literatura académica sobre enseñanza de las ciencias en el primer tercio del siglo xx (Moreno Martínez, 2020a) permite situar a Faraday en un contexto de profunda renovación de la enseñanza de las ciencias. En esta renovación jugó un papel clave el profesorado normalista de ciencias, docentes encargados de formar en ciencias a los futuros maestros. La existencia de instituciones (las Escuelas Normales), un colectivo reglado (los docentes normalistas), una formación específica (los estudios de la Escuela de Estudios Superiores del Magisterio) y órganos de comunicación propios (como Revista de Escuelas Normales o Faraday) muestran la didáctica de las ciencias como un campo profesional claramente conectado en intereses y objetivos con la disciplina actual.

Esa conexión es precisamente la que ha permitido transitar al segundo estadio metodológico: la mirada diacrónica a cuestiones didácticas actuales. En consonancia con los trabajos de Rudolph (2019), el estudio diacrónico de cuestiones didácticas actuales ha de implicar un ejercicio de análisis que evite asumir un contexto educativo como consecuencia directa, lógica y esperable del anterior. En contextos pasados, muchos futuros fueron plausibles, quedando fuera de nuestro presente y, por tanto, del estado de la disciplina actual. Es por ello por lo que la mirada diacrónica permite enriquecer el conocimiento sobre la disciplina didáctica actual al incorporar voces y problemáticas que quedaron excluidas de las genealogías disciplinares. En el presente trabajo, el estudio de las relaciones entre historia y didáctica de las ciencias ha sido la cuestión didáctica actual que ha guiado el análisis realizado.

Finalmente, el tercer estadio metodológico ha permitido transitar del análisis diacrónico de las relaciones entre historia y didáctica en el pasado al análisis epistemológico de la disciplina didáctica en el presente. En este terreno mestizo entre historia y didáctica de las ciencias ha sido especialmente relevante la «apertura interdisciplinar» a la que se aludía en líneas anteriores. Así, al no haberse abordado todavía una sociogénesis panorámica de la didáctica de las ciencias experimentales en España, el análisis realizado se ha nutrido de las perspectivas analíticas empleadas por Mainer Baqué (2009) en su amplio estudio sobre la forja profesional de la didáctica de las ciencias sociales en España. En su análisis, el autor piensa esta didáctica específica como consecuencia de complejos procesos históricos (en absoluto lineales) que establecieron jerarquías y exclusiones en la toma de decisiones sobre la enseñanza y el aprendizaje de las ciencias sociales. Esta mirada crítica ha resultado de especial interés para conectar el análisis de Faraday con otro ineludible reto de la didáctica de las ciencias experimentales en la actualidad: el largo camino entre reflexión didáctica y práctica docente en la enseñanza de las ciencias o lo que es lo mismo, las relaciones entre la didáctica de las ciencias experimentales como disciplina académica y el profesorado de ciencias.

\section{FARADAY: EDITOR, OBJETIVOS, PÚBLICOS Y ESTRUCTURA}

Faraday nació del empeño de uno de los más destacados actores de la enseñanza de las ciencias en España durante el primer tercio del siglo xx, el profesor Modesto Bargalló Ardévol (1894-1981), cuya trayectoria profesional ha sido recuperada y analizada por recientes investigaciones históricas (Moreno Martínez, 2020b, 2021c). Su fundación en enero de 1928 y su publicación hasta al menos mayo de 1929 no puede entenderse separada del quehacer profesional de su editor, quien lo fue también de otra publicación clave en el contexto pedagógico de las escuelas normales de la época: la Revista de Escuelas Normales, un órgano de expresión que fue fundamental para la comunicación entre el profesorado normalista -formadores de docentes- durante las décadas de 1920 y 1930 (Díez Torre et al., 1988). Bargalló participó de su fundación en 1922 como Boletín de Escuelas Normales y fue director de la 
rebautizada Revista desde 1923 hasta 1927 y entre 1931 y 1932. En retrospectiva, podemos entender Faraday como un proyecto nacido de la dilatada tradición como editor de Modesto Bargalló y como una publicación que rivalizó en públicos con la Revista de Escuelas Normales.

Rodolfo Llopis, quien tomó el relevo de Modesto Bargalló como director de la Revista de Escuelas Normales en 1928, se refería así a Faraday como una publicación que tenía por objetivo "coadyuvar a realizar el afán que nuestro profesorado siente por los problemas de la didáctica de los conocimientos fisicoquímicos tan íntimamente ligados con su génesis» (Llopis, 1928, p. 80). Las palabras de Llopis reflejan literalmente la apuesta del recién creado boletín por potenciar la relación entre historia y didáctica de las ciencias, con especial atención a la física y la química. El mismo objetivo aparece recogido en el editorial con que Bargalló iniciaba el número de enero de 1928 de Faraday. Además, el editor invitaba a los profesores «que sientan la bienhechora necesidad de exponer a la consideración de los demás los resultados y las dudas en la labor a que nos debemos» a compartir en las páginas de Faraday «sus trabajos de índole didáctica». Al profesorado normalista de ciencias, que comparte con el didacta actual la formación en ciencias de los futuros docentes, Bargalló añadía otros colectivos profesionales, como el profesorado de enseñanzas técnicas y de institutos de segunda enseñanza, vinculados a la formación de «alumnos de enseñanzas medias» (Bargalló, 1928a, p. 1). Profesores normalistas, maestros de escuela y profesores de enseñanza secundaria y enseñanzas técnicas se convertían así en los potenciales públicos lectores a los que Faraday destinaba sus páginas, tratando de implicar al profesorado de ciencias de las distintas enseñanzas en el debate metodológico sobre cómo enseñar ciencias. Páginas que estaban organizadas en cuatro secciones, cuyos títulos pueden observarse en la cubierta del boletín (figura 1): «Historia, Didáctica, Información y Profesorado». El propio nombre de la revista en honor al célebre científico británico Michael Faraday, y los elementos decorativos de la cubierta, incluyendo el famoso experimento del mercurius praecipitatus per se realizado en la década de 1770 por Antoine Laurent Lavoisier y sus colaboradores, ya anticipan la importancia que tendrá la historia de la ciencia en esta publicación científico-pedagógica.

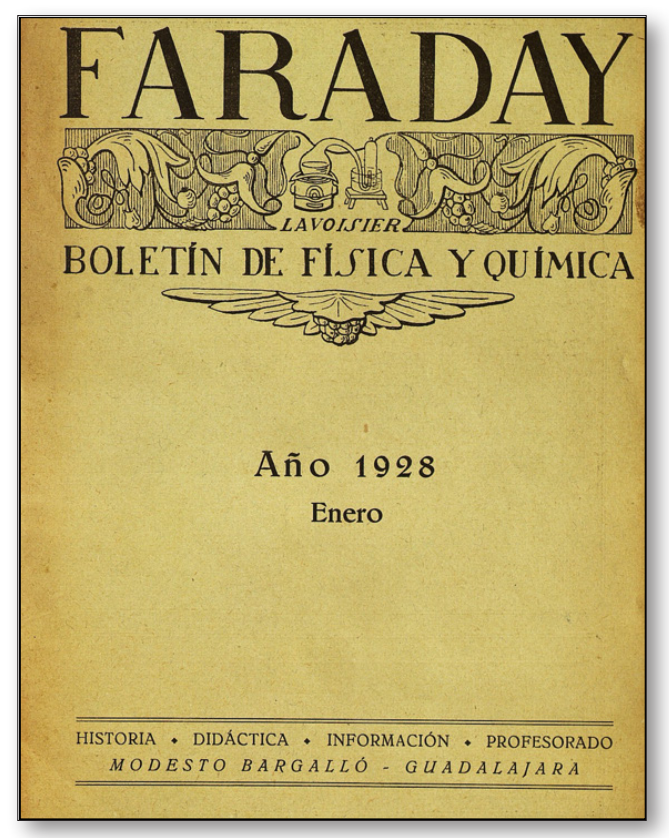

Fig. 1. Cubierta del primer número de Faraday publicado en enero de 1928. 
Los contenidos de la sección Didáctica se recogen en la tabla 2, los cuales fueron elaborados tanto por Bargalló como por otros docentes como Ernesto Caballero, profesor del Instituto de Segunda Enseñanza de Pontevedra; Francisco Manuel Nogueras, profesor de la Escuela Normal de Maestros de Tarragona, y Tomás de la Rica, vinculado a las enseñanzas técnicas como químico de La Hispano en Guadalajara. Este aspecto ilustra la diversidad de colectivos docentes invitados a tomar parte de los debates y reflexiones sobre didáctica de las ciencias promovidos por Faraday. Como puede observarse, se trata de artículos que versaban mayoritariamente sobre diversos aspectos de la enseñanza de la física y de la química, pero también sobre ciencias naturales y sobre problemáticas específicas del profesorado normalista de ciencias. La sección Didáctica solapaba así con las temáticas de algunos editoriales en los que se discutía sobre cuestiones curriculares que implicaban al profesorado de las escuelas normales, como los cambios en los planes de estudio, los concursos de traslado o los procesos de oposición al magisterio. Esto revela otro objetivo subyacente en Faraday aunque no explicitado en la declaración de intenciones inicial: la de servir como órgano de expresión y comunicación entre el profesorado normalista de ciencias, rivalizando claramente con la Revista de Escuelas Normales. Este aspecto se refuerza al examinar la sección Profesorado, donde se incluía información sobre el colectivo normalista en España, como nombramientos, jubilaciones y necrológicas.

Tabla 2.

Artículos publicados en la sección Didáctica del boletín Faraday (1928-1929)

\begin{tabular}{|c|c|}
\hline Número & Titulo (autor) \\
\hline \multicolumn{2}{|r|}{1928} \\
\hline Enero & Ante una posible reforma de Normales. Programas de Física y Química (Modesto Bargalló) \\
\hline Febrero & Algunas minucias en la enseñanza de la física (Modesto Bargalló) \\
\hline Marzo & $\begin{array}{l}\text { Espectros magnéticos (Ernesto Caballero) y Un pequeño perfeccionamiento al aparato de Silber- } \\
\text { mann empleado en la reflexión de la luz (Francisco Manuel Nogueras) }\end{array}$ \\
\hline Abril & Desconocido \\
\hline Mayo & Enseñanzas de la práctica. Las cualidades de los aceros (Tomás de la Rica) \\
\hline Septiembre & Nota sobre la enseñanza de la mineralogía (Francisco Manuel Nogueras) \\
\hline Octubre & Aparato para demostrar las leyes del tornillo, torno y polea (Francisco Manuel Noguera) \\
\hline Nov. - Dic. & Algunas minucias en la enseñanza de la física II (Modesto Bargalló) \\
\hline \multicolumn{2}{|r|}{1929} \\
\hline Enero & $\begin{array}{l}\text { Reformas en la nomenclatura química según la novena Conferencia Internacional de Química. } \\
\text { Resumen de los extractos publicados en Anales de la Sociedad Española de Física y Química y de } \\
\text { la Revista Catalana Ciència, tomados del Bulletin de la Société Chimique de France (Modesto } \\
\text { Bargalló) }\end{array}$ \\
\hline Febrero & $\begin{array}{l}\text { Al margen de las oposiciones al magisterio. La enseńanza de las ciencias físicas (Modesto Bar- } \\
\text { galló) }\end{array}$ \\
\hline Marzo & $\begin{array}{l}\text { Ante la anunciada reforma del plan de las Normales. Las ciencias físico-químicas y naturales } \\
\text { (Modesto Bargalló) }\end{array}$ \\
\hline Abril & $\begin{array}{l}\text { Ante la reforma de las Escuelas Normales. El material de enseñanza de las Escuelas Normales } \\
\text { (Modesto Bargalló) }\end{array}$ \\
\hline Mayo & Los exámenes libres de Física y Química (Modesto Bargalló) \\
\hline
\end{tabular}

Los contenidos de la sección Historia se recogen en la tabla 3. Estos también dan cuenta de otro objetivo clave de Faraday: traducir al castellano breves fragmentos de textos clásicos de la historia de la 
ciencia para hacerlos accesibles al profesorado. Los artículos de la serie «Las doctrinas fundamentales de la química" y "Las experiencias clásicas de la física» estaban conformados por extractos de varias obras de Isaac Newton, John Dalton o Amadeo Avogadro, entre otros. La importancia de disponer de colecciones de textos clásicos de la historia de la ciencia fue otra empresa por la que Bargalló abogó a través de las páginas de Faraday. Una herramienta de especial interés para ello fue la sección Información, donde recopiló títulos de varias obras sobre historia de la ciencia publicadas en otros países, denunciando el desinterés de las instituciones españolas. Esta sección, como veremos, también albergaba información relevante sobre instituciones, revistas y congresos de historia de la ciencia de diversos países. Bargalló pretendía así hacer circular información sobre la emergente comunidad internacional de historiadores de la ciencia y señalar su escasa presencia en España, donde «solo Faraday con su extremada modestia, sin auxilio de nadie» se ocupaba «con asiduidad» de «textos y problemas» de la historia de la ciencia (Bargalló, 1928b, p. 1).

Tabla 3.

Artículos publicados en la sección Historia del boletín Faraday (1928-1929)

\begin{tabular}{|c|c|}
\hline Número & Titulo \\
\hline \multicolumn{2}{|r|}{1928} \\
\hline Enero & Las experiencias clásicas de la Física I. La Óptica de Isaac Newton (1704) \\
\hline Febrero & Las doctrinas fundamentales de la Química. Teoría atómicomolecular I. La labor de John Dalton \\
\hline Marzo & Las experiencias clásicas de la Física II. La Óptica de Isaac Newton (1704) \\
\hline Abril & $\begin{array}{l}\text { Desconocido, pero posiblemente se trató de: Las doctrinas fundamentales de la química. Teoría atómico- } \\
\text { molecular II }\end{array}$ \\
\hline Mayo & Las doctrinas fundamentales de la química. Teoría atómicomolecular III \\
\hline Septiembre & Las doctrinas fundamentales de la química. Teoría atómicomolecular IV. Labor de Ampere y de Gaudin \\
\hline Octubre & $\begin{array}{l}\text { El diario de Faraday. La experiencia del Puente de Waterloo. Extracto de The Observer enviado por la } \\
\text { profesora Doña Bárbara Aitken. «Valor del estudio de los clásicos de la ciencia» firmado por L. Poincaré } \\
\text { (conferencia del Museo Pedagógico de París, 1904). }\end{array}$ \\
\hline $\begin{array}{l}\text { Noviembre- } \\
\text { Diciembre }\end{array}$ & $\begin{array}{l}\text { Las experiencias clásicas de la Física. La «óptica» de Isaac Newton (1704) III. Separación de los rayos } \\
\text { heterogéneos de la luz compuesta }\end{array}$ \\
\hline \multicolumn{2}{|r|}{1929} \\
\hline Enero & \multirow[t]{2}{*}{ La pequeña máquina electromagnética de Pacinotti (1860). Extracto de Nuevo Cimento (1864) } \\
\hline Febrero & \\
\hline Marzo & La organización de los historiadores de la ciencia. Extracto de Coopération Intellectuelle, por Aldo Mieli \\
\hline Abril & Las experiencias clásicas de la Física IV. La Óptica de Newton (1704) \\
\hline Mayo & $\begin{array}{l}\text { Las experiencias clásicas de la Física. Acción de la corriente sobre la aguja imantada: Experiencia de Oers- } \\
\text { ted y regla de Ampere (1820) }\end{array}$ \\
\hline
\end{tabular}

\section{FARADAY: UNA VENTANA A LAS RELACIONES ENTRE HISTORIA Y DIDÁCTICA DE LAS CIENCIAS}

El análisis de Faraday desde el marco historiográfico y metodológico detallado al inicio del presente trabajo revela esta publicación como una ventana privilegiada para explorar las relaciones entre historia y didáctica de las ciencias en la España del primer tercio del siglo xx. En este periodo tuvo lugar la emergencia y paulatina consolidación disciplinar de la comunidad internacional de historiadores de la ciencia (Bertomeu Sánchez y García Belmar, 2008). Todo ello se plasmó en la creación de instituciones 
dedicadas a la investigación histórica sobre ciencia, la producción de trabajos escritos y la celebración de congresos. Las páginas de Faraday se hicieron eco de todos estos aspectos. Así, parapetado en un plural mayestático, Bargalló escribía en el editorial del número de octubre de 1928 que:

Uno de los fines que nos propusimos al iniciar nuestra publicación [Faraday] fue estimular el interés del profesorado hacia la historia de las ciencias fisicoquímicas. Nuestro país no se ha interesado como debe por las investigaciones sobre la génesis de la ciencia; debido, tal vez en parte, a que no ha existido acicate de descubrir ningún oculto tesoro científico nacional, creencia que pudiera pecar de exagerado pesimismo. Urge, como han hecho otras naciones, crear un Instituto dedicado a la Historia de las Ciencias, cuyo estudio tanto habrían de contribuir a completar la propia Ciencia (Bargalló, 1928c, p. 1).

Faraday apostaba así por la visibilidad de los estudios sobre historia de la ciencia en la comunidad académica española. Se trata de un argumentario defendido por algunos intelectuales de la época, como Francisco Vera Fernández de Córdoba, uno de los impulsores de la Asociación Española de Historiadores de la Ciencia que se creará en 1934, o Eugenio D’Ors (Cobos Bueno, 2003). Este último había expresado unos ańos antes del nacimiento de Faraday que convenía «dar amplio estado universitario» a la historia de la ciencia, afirmando que:

Siempre he pensado que unos cuantos ańos de calor dado a los mismos [los estudios de la historia de la ciencia] de cuidado en su eficacia psicológica y social, tendría virtud de mudar para siempre la faz científica de Espańa... No menos ha alcanzado, en su orden respectivo, los estudios de historia de la literatura o de historia del arte (D’Ors, 1925, p. 8).

Todos estos aspectos reflejan cierto interés por parte de algunos intelectuales de la época en la institucionalización de la historia de la ciencia. No obstante, Faraday también mostraba la existencia de algunas posturas entre el desdén y el desinterés. Así, Bargalló empleaba su editorial en el número de mayo de 1928 para tratar de excusar las palabras del entonces presidente de la Sociedad Espańola de Física y Química, Julio Palacios, en el acto de las bodas de plata de dicha institución. Tras destacar la labor de algunos científicos españoles como Andrés Manuel del Río o los hermanos Delhuyar, el eminente físico señaló en su discurso que «la gloria científica» había que buscarla «en los laboratorios y no en los archivos»; a lo que Bargalló respondía a través de Faraday y, de nuevo, refugiado en un plural mayestático, que «sentiríamos que, amparándose en la autoridad de su autor y no desgajándola del discurso, se diera a esta frase un valor que no tiene» (Bargalló, 1928d, p. 1). En esta línea, alabó que otros países hubiesen publicado «obras admirables, fundado revistas y hasta Escuelas e Institutos especiales de investigación en Historia de las Ciencias» (Bargalló, 1928d, p. 1).

Faraday, una publicación nacida por y para el profesorado de escuelas de primera enseñanza, escuelas normalistas, institutos y escuelas técnicas, se revela de este modo como una herramienta para la difusión de la institucionalización de la historia de la ciencia durante un periodo efervescente para esta disciplina histórica. El boletín difundió abundante información sobre colecciones de obras, publicaciones, congresos e instituciones de historia de la ciencia de diferentes países. Estas referencias serán frecuentes desde el primer número de Faraday, como ilustran las siguientes líneas:

Hace dos años el Instituto Nacional Italiano para la Historia de las Ciencias Físicas y Matemáticas publicaba bajo la dirección de Federico Enriques una colección de obras sobre Historia de las Ciencias Matemáticas y Físicas. Cumple Italia el desempeño que en Alemania, en proporciones mucho mayores, han tenido los Ostwald's Klassiker; en Francia, en escala más modesta a los Clásicos de Ostwald, las colecciones Les maîtres de la pensé scientifique, y Science et Civilisation, dirigida por Solovine, o Les classiques de la Science, de H. Abraham, Gauthier, Le Chatelier, Lemoine; o en los países de habla inglesa los Mathematical Monographs o los Open Court Classics of Science and Philosophy. ¿Cuándo tendremos en lengua española una colección semejante? (Bargalló, 1928d, p. 10). 
Otro ejemplo de interés lo encontramos en el número de octubre de 1928, en el que se referenciaba la creación reciente de un instituto de historia de la medicina, de las matemáticas y de las ciencias fisiconaturales en Leiden, a lo que se añadía:

Poco a poco todas las naciones crean organismos para cultivar la Historia de las Ciencias: indicio de que es comprendido el gran valor que tiene el conocimiento de la evolución de las disciplinas científicas, para la enseñanza y para el progreso de la cultura, y muchas veces para el honor de las propias naciones (Bargalló, 1928e, p. 10).

Especial mención merece el artículo publicado en el número de marzo de 1929. En la sección Historia Bargalló incluía el extracto de una nota que había sido publicada por Aldo Mieli en Coopération Intellectuelle tan solo un mes antes, como se puede observar en la figura 2. Faraday cedía así sus páginas a uno de los principales actores históricos en el impulso disciplinar a la historia de la química del primer tercio del siglo xx (Bertomeu Sánchez y García Belmar, 2008). También aparecerán recomendados en Faraday los trabajos de George Sarton, pionero de la historia de la ciencia a nivel internacional, incluyendo artículos publicados en la prestigiosa revista Isis.

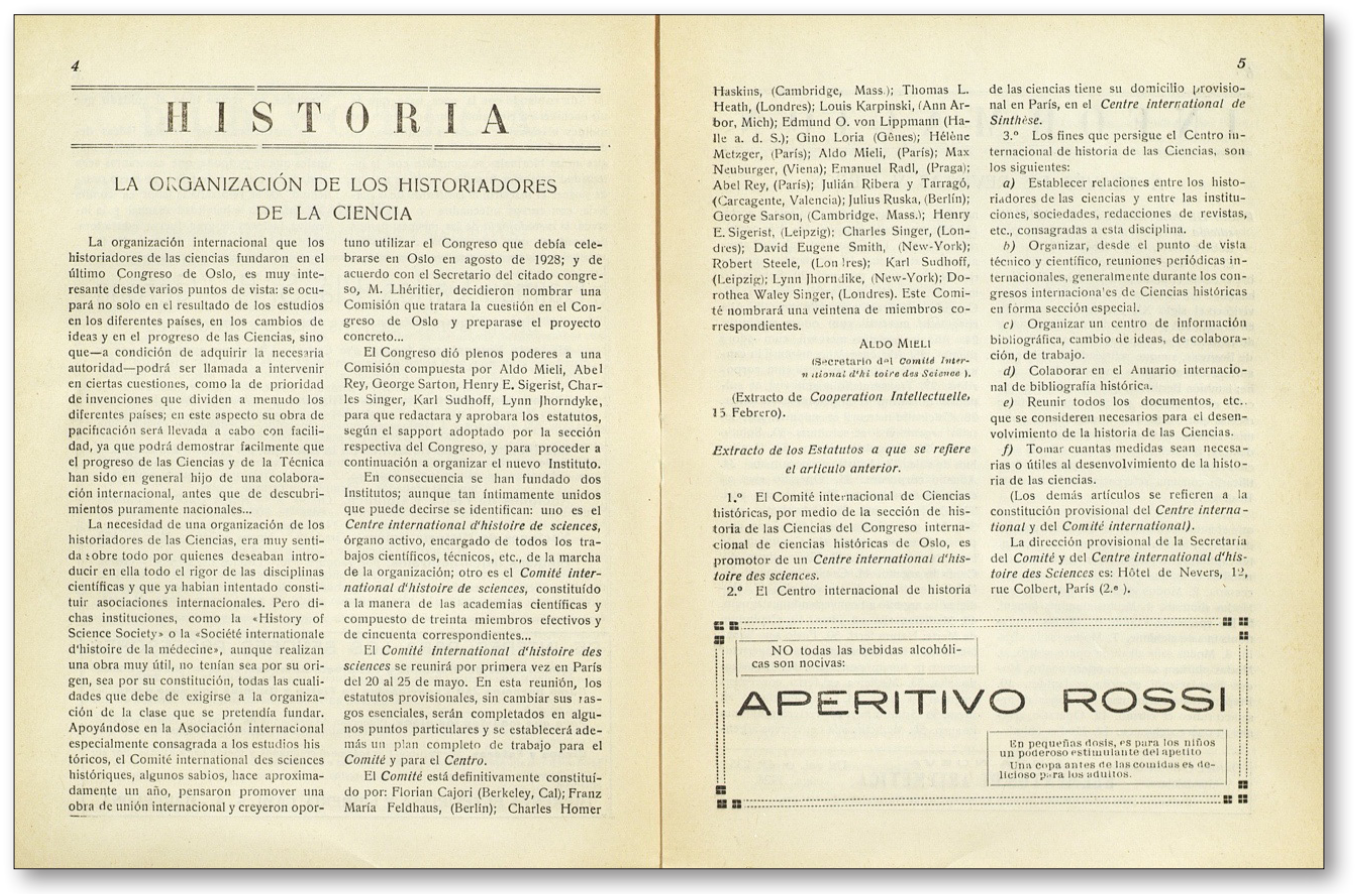

Fig. 2. Sección Historia del boletín Faraday correspondiente al número de marzo de 1929.

Si Bertomeu Sánchez y García Belmar (2008) han mostrado la profunda relación entre historia y didáctica de la química a través del Journal of Chemical Education, Faraday nos ofrece un ejemplo excepcional en este sentido para la historia y la didáctica de las ciencias en el contexto español, pues ilustra cómo la didáctica de las ciencias nutrió de espacios comunicativos y públicos a la historia de la ciencia, lo que propició la circulación de los emergentes productos de su forja disciplinar (instituciones, congresos, órganos de expresión, etc.).

No obstante, la relación entre historia y didáctica de las ciencias en modo alguno puede entenderse como unidireccional. Este aspecto puede colegirse de una de las líneas anteriores en las que Bargalló destacaba «el gran valor que tiene el conocimiento de la evolución de las disciplinas científicas para la enseñanza» (Bargalló, 1928e, p. 10). La historia de la ciencia dotó a la didáctica de las ciencias del 
primer tercio del siglo xx de un fértil andamiaje epistemológico y metodológico. Modesto Bargalló fue una de las principales voces en la defensa de la historia en la didáctica de las ciencias en Espańa y en la propia demarcación de la didáctica de las ciencias en el seno de la reflexión pedagógica. En esta línea, una década antes de la fundación de Faraday, este maestro de maestros catalán escribía en su lengua natal:

No caiguem en la ridiculesa de voler posseir una formació pedagògica sense tenir contingut per a formar. El coneixement fonamental de l'evolució d'una ciència porta en si mateix el mètode, el pla d'ensenyament, que no es pot confondre de cap manera amb l'art, la gràcia d'ensenyar, que tampoc s'aprèn per mitjà d'estudis pedagògics (Bargalló, 1918, p. 219).

Bargalló delimitaba así la didáctica de las ciencias como campo en el terreno pedagógico en el que el conocimiento de la historia de las ciencias resultaba fundamental. Por ello, se requería formar en historia de la ciencia a los futuros docentes. Así, en su conferencia en la Escola d'Estiu de Barcelona de 1930, cuyo contenido se publicó en forma de libro dos años después, Bargalló hacía una petición al profesorado asistente:

Yo os pido que cuando tengáis en la enseñanza de las ciencias alguna duda de orden metodológico, no busquéis solamente una solución en el buen libro de Didáctica: consultad también un buen manual de Historia de la Cultura o de la Ciencia respectiva y tal vez tengáis inspiraciones no sospechadas (Bargalló, 1932, p. 12).

El proyecto pedagógico de Bargalló como docente normalista ha sido objeto de análisis detallado recientemente (Moreno Martínez, 2020b, 2020c). Dicho proyecto cimentó las prácticas pedagógicas y la cultura material del aula de ciencias sobre las fases que, de acuerdo con una concepción comteniana de la historia de la ciencia, habrían atravesado las disciplinas científicas en el tiempo. Si de acuerdo con el pensamiento del filósofo francés Auguste Comte la historia de las ciencias habría transitado por una fase netamente observacional (cualitativa), una fase experimental (cuantitativa) y una fase en la que el conocimiento científico se estructuraba en leyes y teorías; la ciencia en las aulas debía partir de la observación, para después introducir la experimentación y solo en los niveles superiores, el estudio de leyes y teorías científicas. Este «alto valor» de la historia de la ciencia para inspirar la metodología didáctica se apoyaba en el estudio del «desenvolvimiento mental» del niño, promovido por diferentes filósofos y pedagogos en el marco de la entonces llamada paidología (Pozo Andrés, 2003). Esta profunda imbricación entre ontogenia (el desarrollo del discente) y filogenia (el desarrollo del conocimiento científico) será compartida por educadores de distintos países, auspiciados desde finales del siglo XIX por la conocida como cultural epoch theory de Herbert Spencer (Rudolph, 2019), y fue reapropiada activamente por el célebre epistemólogo Jean Piaget en la década de 1980 (Piaget y García, 1982).

A fin de poder cimentar la didáctica de las ciencias sobre el conocimiento de su génesis y desarrollo histórico, se erigía fundamental dotar al profesorado encargado de formar a los futuros maestros -el profesorado normalista- de herramientas materiales e intelectuales sobre historia de la ciencia. Desde Faraday se asumió esta tarea. Los diferentes textos históricos incluidos por Bargalló en el boletín dan cuenta de ello. Así, por ejemplo, en la sección Didáctica del número de noviembre y diciembre de 1928 Bargalló recomendaba al profesorado de ciencias definir el movimiento uniforme en base al Traité de Dynamique de D'Alembert, donde se definía como «aquel que no fuese modificado por fuerza alguna» (Bargalló, 1928f, p. 3). Se trata de una recomendación que Bargalló defendía con frecuencia en muchas de sus obras para maestros y manuales de ciencias, argumentando que «nadie tan anheloso en describir experiencias y en exponer las leyes con claridad y sencillez que en general lo han hecho los fundadores de la ciencia» (Bargalló, 1929, nota inicial). La historia de la ciencia aportaría así a la didáctica de las ciencias una mayor claridad a la hora de presentar los conceptos estructurantes de las ciencias 
escolares. Bargalló, en la línea defendida entonces por el filósofo de la ciencia Jules Henry Poincaré, destacaba el valor de los textos clásicos en la enseñanza de las ciencias frente a las presentaciones didácticas de muchos manuales que «se dicen elementales», pero en los que «los autores contadas veces llegan a las fuentes, y que copiándose a menudo unos de otros han reproducido con deformaciones cada vez más señaladas las ideas primitivas de los inventores» (Bargalló, 1932, p. 19). Faraday respondió a esta necesidad didáctica produciendo textos clásicos que proporcionaban al profesorado-lector una plétora de referencias históricas para enseñar conceptos fundamentales de las ciencias, como átomo y molécula a través de textos de Dalton y Avogadro o conceptos básicos de óptica mediante textos de Newton.

El análisis de Faraday ha permitido identificar otro uso didáctico históricamente desempeñado por la historia de la ciencia en la enseñanza. Se trata de su papel para motivar al alumnado y promover una serie de valores sobre la ciencia como empresa intelectual. Este aspecto queda patente en el propio nombre del boletín. Que Faraday tomara su nombre del célebre científico británico no fue en modo alguno una decisión arbitraria. El propio Bargalló explicaba la elección en el editorial del primer número:

Véase en el nombre FARADAY que hemos adoptado, un símbolo que nos guía. Sirvan de norma la vida y la obra del sabio inglés. Sencilla y templada, impregnada por la avidez de superarse con los escasos medios de que disponía, aprendiendo siempre, solitario, sin maestros, con el noble empeño de saciase en todas las ramas del espíritu; y con el admirable tesón de que está poseído quien confía vencer en la lucha [...]. Hombres de esta naturaleza son los que verdaderamente educan: aquellos cuyo espíritu une al genio una voluntad pura, tenaz y cordial (Bargalló, 1928a, p. 1).

En un contexto de escasa tradición de las ciencias en la escuela primaria y de deficitaria formación científica del profesorado, la didáctica de las ciencias encontró en la historia de la ciencia una valiosa aliada para educar y motivar. Faraday nos revela así una profunda imbricación entre historia y didáctica de las ciencias solamente comprensible desde una concepción de relación bidireccional y estrecha entre ambas, que supera las lindes disciplinares que hoy las separan.

\section{CONCLUSIONES Y COMENTARIO FINAL}

A tenor del análisis del boletín Faraday presentado en las líneas anteriores es posible colegir con claridad la importancia que la historia de la ciencia ha tenido para la didáctica de las ciencias. Facilitar la enseñanza de los conceptos estructurantes de la ciencia escolar, promover valores, motivar al alumnado y propiciar el diseño de prácticas pedagógicas eficaces son algunos de los usos didácticos que los docentes de ciencias y los docentes encargados de su formación (el profesorado normalista) encontraron en la historia de la ciencia. De este modo, el interés de la historia para enseñar ciencias en modo alguno puede entenderse como una consecuencia del constructivismo piagetiano heredado por la actual disciplina didáctica. La didáctica de las ciencias, ya fuese como actividad profesional o como disciplina académica, ha encontrado en la historia de la ciencia una fuerza motriz para su desarrollo. Faraday lo muestra poniendo de manifiesto una interconexión hasta ahora poco o nada transitada: la vinculación entre la forja disciplinar del colectivo de historiadores de la ciencia y la renovación pedagógica de la enseñanza de las ciencias del primer tercio del siglo xx. Ambos procesos históricos confluyeron en las páginas de Faraday, reforzando así el papel de la historia de la ciencia como herramienta intelectual de la didáctica de las ciencias. De este modo, su uso fue más allá del aporte de ciertas biografías para el aula o de definiciones más claras para entender conceptos de física y química. Además, la historia de la ciencia dotó a la didáctica de las ciencias de una plétora de concepciones epistemológicas y metodológicas sobre la que se estructuró el diseño de estrategias de enseñanza-aprendizaje. Se trata de un aspecto clave al no contar entonces la didáctica de las ciencias de un andamiaje epistemológico propio. Al mismo tiempo, las páginas de Faraday han ilustrado cómo la discusión en torno a cuestiones sobre 
didáctica de las ciencias propició la circulación de obras, problemáticas e instituciones que entonces comenzaban a edificar a la disciplina histórica.

Si bien la consolidación disciplinar de la historia y la didáctica de las ciencias ha tenido múltiples beneficios para ambas, también ha introducido desconexiones entre las respectivas comunidades académicas, pese a que, tal y como ha mostrado el análisis de Faraday, ambas se desarrollaron con puntos de contacto y notables sinergias en el pasado. El examen de Faraday permite así ofrecer otro elemento de reflexión epistemológica sobre la didáctica de las ciencias experimentales: su vínculo con el docente de ciencias. El colectivo normalista, encargado de formar en ciencias a los futuros maestros y maestras y en cuyo seno nació Faraday, tuvo que gestar su identidad rivalizando con otros colectivos con los que al mismo tiempo debía colaborar. Así, en las páginas de Faraday coincidieron docentes de escuela primaria -educandos de los docentes normalistas- y docentes de enseńanza secundaria, con quienes el profesorado normalista rivalizó ante los cambios legislativos y normativos que llegaron a llevar parte de la formación de los futuros docentes a los institutos. Todos estos colectivos constituyeron el público lector que condicionó la elaboración de los contenidos de Faraday. Esta situación, si bien con importantes limitaciones (como la ausencia de estatus universitario para el magisterio), revela no obstante una mayor horizontalidad y pluralidad entre colectivos docentes implicados activamente en los debates sobre enseñanza y aprendizaje de las respectivas disciplinas escolares. Este aspecto, ya señalado para la didáctica de las ciencias sociales, ha sido perfectamente identificable en Faraday para el ámbito de la enseñanza de las ciencias físicas, químicas y naturales. Este "periodo adisciplinar» de la didáctica de las ciencias experimentales se revela como una oportunidad para pensar también las limitaciones de los procesos de conformación disciplinar y la necesidad de propiciar una mayor reflexión epistemológica sobre la disciplina basada en las nuevas perspectivas historiográficas y resultados de investigación histórica sobre ciencia en las aulas. Estas nuevas miradas permitirán recuperar personajes e iniciativas -como Bargalló y Faraday - que contribuyan a humanizar y contextualizar la enseñanza de las ciencias como actividad profesional y a fomentar una mayor colectividad entre los distintos profesionales vinculados a la educación científica, contribuyendo así a acortar la distancia entre investigación en didáctica de las ciencias experimentales y práctica docente.

\section{AGRADECIMIENTOS}

La investigación en que se inscribe el presente artículo se realizó en el marco de una Beca de la Fundación Juanelo Turriano a Tesis Doctorales en Historia de la Ciencia y fue ampliada gracias a una Beca de Excelencia Postdoctoral del Gobierno de México.

\section{REFERENCIAS BIBLIOGRÁFICAS}

Adúriz Bravo, A. (2000). La didáctica de las ciencias como disciplina. Enseñanza, 17-18, 61-74.

Adúriz Bravo, A. e Izquierdo Aymerich, M. (2002). Acerca de la didáctica de las ciencias como disciplina autónoma. Revista Electrónica de Enseñanza de las Ciencias, 1(3), 130-140.

Bargalló, M. (1918). Preliminars a l'ensenyament de la química. Quaderns d'estudi, 2, 216-221.

Bargalló, M. (1928a). Propósitos. Faraday, I(1), 1.

Bargalló, M. (1928b). Los clásicos de la ciencia. Faraday, I(8), 1.

Bargalló, M. (1928c). La evolución de las ciencias. Faraday, $I(7), 1$.

Bargalló, M. (1928d). Información. Faraday, I(1), 10.

Bargalló, M. (1928e) Información. Faraday, II(7), 10.

Bargalló, M. (1928f). Algunas minucias en la enseñanza de la física II. Faraday, I(8), 2-3. 
Bargalló, M. (1929). Manual de Física (2. a ed.). Reus: Ediciones Sardá.

Bargalló, M. (1932). La enseñanza experimental en la escuela. Su relación con el desarrollo histórico de la física y de la química. Reus: Ediciones Sardá.

Bernal Martínez, J. M. (2001). Renovación pedagógica y enseñanza de las ciencias. Medio siglo de propuestas pedagógicas y experiencias escolares (1882-1936). Madrid: Biblioteca Nueva.

Bertomeu Sánchez, J. R. (2016). Beyond borders in the History of Science Education. En T. Arabatzis, J. Renn y A. Simoes (Eds.), Relocating the History of Science. Essays in Honor of Kostas Gavroglu (pp. 159-173). Dordrecht: Springer.

Bertomeu Sánchez, J. R. y García Belmar, A. (2008). La historia de la química: Pequeña guía para navegantes. Parte I: Viejas y nuevas tendencias. Anales de Quimica, 104(1), 56-63.

Burke, P. (2017). ¿Qué es la historia del conocimiento? Cómo la información dispersa se ha convertido en saber consolidado a lo largo de la historia. Buenos Aires: Siglo XXI.

Checa Godoy, A. (2002). Historia de la prensa pedagógica en España. Sevilla: Publicaciones de la Universidad de Sevilla.

Cobos Bueno, J. M. (2003). La Asociación Española de Historiadores de la Ciencia: Francisco Vera Fernández de Córdoba. Llull, 26, 57-81.

Díez Torre, A. R., Pozo Andrés, M. M. del y Segura Redondo, M. (1988). La Revista de Escuelas Normales: Una publicación de regeneración normalista nacida en Guadalajara (1923-1936). Revista Interuniversitaria de Formación del Profesorado, 1, 9-29.

D’Ors, E. (3 de diciembre de 1925). $A B C$, p. 8.

Gavroglu, K. y Simoes, A. (2016). Philosophical issues in (sub)disciplinary contexts. The case of Quantum Chemistry. En E. Scerri y G. Fisher (Eds.), Essays in the Philosophy of Chemistry (pp. 6079). Nueva York: Oxford University Press.

Hannaway, O. (1975). The Chemists and the World: The Didactic Origins of Chemistry. Estados Unidos: John Hopkins University Press.

Izquierdo Aymerich, M. (2011). Historia de la química y enseñanza de la química. En A. Caamaño (coord.), Física y Química. Complementos de Formación Disciplinar (pp. 53-73). Barcelona: Graó.

Kaiser, D. (2005). Pedagogy and the practice of science. Historical and Contemporary Perspectives. Londres: MIT Press.

Kragh, H. (2007). Una introducción a la historia de la ciencia. Barcelona: Crítica.

Llopis, R. (1928). Prensa y noticias. Revista de Escuelas Normales, 51, 80.

Mainer Baqué, J. (2009). La forja de un campo profesional. Pedagogía y didáctica de las ciencias sociales en España (1900-1970). Madrid: Consejo Superior de Investigaciones Científicas.

Montes Moreno, S. (2003). La Escuela Moderna. Revista pedagógica hispanoamericana (1891-1934). Barcelona: Pomares.

Moreno Martínez, L. (2020a). Ciencia en las aulas: actores, espacios, prácticas pedagógicas y cultura material de la enseñanza de las ciencias en España durante el primer tercio del siglo xx. Dynamis, $40(1), 225-235$.

Moreno Martínez, L. (2020b). Ciencia en las aulas: Prácticas pedagógicas, cultura material e historia de la ciencia en la obra de Modesto Bargalló en España (1894-1939). Valencia: Universitat de València.

Moreno Martínez, L. (2020c). Modesto Bargalló en España (1894-1939). Una biografía entre la historia de la educación y la historia de la ciencia. Historia y Memoria de la Educación, 13, pp. 635-674.

Navarro Brotons, V. (1983). La historia de las ciencias y la enseñanza. Enseñanza de las ciencias, 1(1), 50-53.

Nieto-Galan, A. (2011). Los públicos de la ciencia. Expertos y profanos a través de la historia. Barcelona: Marcial Pons. 
Nieto-Galan, A. (2014). Las «historias de la ciencia» y sus adaptaciones a la enseñanza: un debate abierto. En M. Quintanilla Gatica, S. Daza Rosales y H. G. Cabrera Castillo (Eds.), Historia y filosofía de la ciencia. Aportes para una "Nueva aula de ciencias", promotora de ciudanía y valores (pp. 66-75). Santiago de Chile: Bellaterra.

Nye, M. J. (1993). From Chemical Philosophy to Theoretical Chemistry. Dynamics of matter and dynamics of disciplines, 1800-1950. California: University of California Press.

Olesko, K. (2006). Science Pedagogy as a Category of Historical Analysis: Past, Present, and Future. Science \& Education, 15, 863-880.

Olesko, K. (2014). Science Education in Historical Study of Sciences. En M. Matthews (Ed.), International Handbook of Research in History, Philosophy and Science Teaching (pp. 1965-1990). Ámsterdam: Springer.

Piaget, J. y García, R. (1982). Psicogénesis e historia de la ciencia. México: Siglo XXI Editores.

Porlán Ariza, R. (1998). Pasado, presente y futuro de la didáctica de las ciencias. Enseñanza de las ciencias, 16(1), 175-185.

Pozo Andrés, M. M. del (2003). La renovación pedagógica de los métodos de enseñanza: El movimiento de la escuela nueva. En G. Ossenbach Sauter (Coord.), Psicología y pedagogía en la primera mitad del siglo XX (pp. 43-72). Madrid: UNED.

Quintanilla Gatica, M., Daza Rosales, S. y Cabrera Castillo, H. (Eds.) (2014). Historia y filosofía de la ciencia. Aportes para una "nueva aula de ciencias", promotora de ciudadanía y valores. Santiago de Chile: Bellaterra.

Rudolph, J. L. (2019). How we teach Science? What's changed and why it matters. Cambridge, Massachusetts: Harvard University Press.

Simon, J. y Herrán, N. (Eds.) (2008). Beyond Borders. Fresh Perspectives in History of Science. Newcastle: Cambridge Scholars Publishing.

Viñao, A. (2004). Escuela para todos. Educación y modernidad en la España del siglo XX. Madrid: Marcial Pons. 


\title{
The bulletin Faraday (1928-29) and the relations between history and didactics of science
}

\author{
Luis Moreno Martínez \\ Instituto Interuniversitario López Piñero, Universitat de València (España) \\ luis.moreno-martinez@uv.es
}

This paper analyses one of the first Spanish journals on the history and didactics of science: Faraday. This journal was founded in 1928 by Modesto Bargalló (1894-1939), who was one of the most outstanding science educators of the first third of the $20^{\text {th }}$ century in Spain. This journal was an important part of his work in Spain, where he developed a successful career as a science teacher educator, editor and author of several science textbooks for primary school teachers, high school teachers and normal school educators.

The unprecedented analysis of Faraday has been designed attending to the fresh perspectives in history of science education. Starting from the contents of this journal, I have studied the relations between the history and didactics of science in the first decades of the $20^{\text {th }}$ century in Spain. This analysis has pointed out that history of science had a key role in the pedagogical renewal of science education during this period. Faraday reveals that history of science provided science teachers with a wide range of epistemological approaches and didactic tools in order to promote new methodologies in science teaching. The use of historical sources and the relations between student development and the history of scientific knowledge were some examples of the role of history of science in teaching.

Likewise, the analysis of Faraday has highlighted that science teaching served as a circulation framework to the international community of historians of science. Thus, many references to journals (like Isis), books, research institutions, conference and authors (like Aldo Mieli and George Sarton) were found in Faraday. I argue that this double relation between history and didactics of science was a key factor in the emergence of history of science as an academic field in Spain. The paper also concludes that history of science had a major role in the methodological reflection on science teaching, even though the disciplinary origin of the didactics of science in Spain has been situated in the last decades of the $20^{\text {th }}$ century.

Finally, it should be noted that, although during the first third of $20^{\text {th }}$ century history and didactics of science were not consolidated academic disciplines, the relations between both fields of knowledge were strong and crucial. Despite of the advantages of the current academic structure for both disciplines, Faraday shows the wide range of possibilities that collaboration between didactics and history of science can offer us in order to improve our current science education and foster a fruitful dialogue between science teachers and researchers in history of science and science education. 